\title{
Perspectiva de género en la formación de profesionales de la salud: U na tarea pendiente
}

\author{
Estela Arcos ${ }^{1 a}$, Johanna Poblete ${ }^{1 a}$, Irma Molina Vega2b, \\ Christian Miranda $3 c$, Yanira Zúñiga ${ }^{4 d}$, Ester Fecci ${ }^{5 e}$, \\ Laura Rodríguez ${ }^{6 f}$, Myriam Márquez ${ }^{19}$, Miguel Ramírez ${ }^{7 h}$. \\ Gender perspective in health care \\ teaching: A pending task
}

Background: Gender must be considered in the design and implementation of health policies to safeguard equity and accomplish sanitary objectives. Aim: To identify gender perspective in the curricula of five health care careers in the Universidad Austral de Chile. To identify the situation of women in the teaching profile of such curricula. Material and methods: An exploratory and descriptive study with a critical reading of the structure of the programs of 217 courses. Revision of official academic registries. Results: Gender is usually not included in the curricula of health care careers. The generic language conceals female academics and students. There was a scarce inclusion of cross sectional issues such as collaborative work, interpersonal and democratic relationship, equity and critical analysis. There were no differences in academic achievements between female and male students. The contractual profile of female academics reproduces the gender inequity of the work market. Conclusions: The inclusion of gender is a pending task in the training of health care professionals (Rev Méd Chile 2007; 135: 708-17).

(Key words: Behavior and behavior mechanisms: Education professional; Gender indentity; Health services)

Recibido el 11 de agosto, 2006. Aceptado el 14 de diciembre, 2006.

Trabajo financiado por Proyecto S-2004-22, Dirección de Investigación y Desarrollo, Universidad Austral de Chile. No hubo influencia en el diseño del estudio; ni en la recolección, análisis o interpretación de los datos.

${ }^{1}$ Instituto de Enfermería Materna, Facultad de Medicina. ${ }^{2}$ Instituto de Estadísticas, Facultad de Ciencias Económicas y Administrativas. ${ }^{3}$ Instituto de Filosofía y Estudios Educacionales, Facultad de Filosofía y Humanidades. ${ }^{4}$ Instituto de Derecho Público, Facultad de Ciencias Sociales y Jurídicas. ${ }^{5}$ Instituto de Administración, Facultad de Ciencias Económicas y Administrativas. ${ }^{6}$ Instituto de Arquitectura y Urbanismo, Facultad de Ciencias de la Ingeniería. ${ }^{7}$ Instituto de Economía, Facultad de Ciencias Económicas y Administrativas. Universidad Austral de Chile. Valdivia, Chile.

aMatrona, Magíster en Desarrollo Rural. bLicenciada en Educación y Master en Estadística.

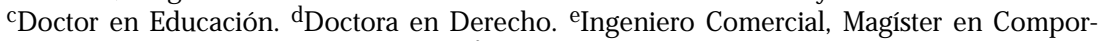
tamiento y Desarrollo Organizacional. fProfesora de Historia y Geografía, Master of Arts in Geography. gMatrona, Magíster en Educación e ${ }^{\mathrm{h}}$ Ingeniero Comercial.

\footnotetext{
Correspondencia a: Estela Arcos, Instituto de Enfermería Materna, Facultad de Medicina, Universidad Austral de Chile. Casilla 567, Valdivia-Chile. Fax: 56-63-293458. E mail: earcos@uach.cl
} 
L a salud de la mujer está vinculada a su posición en la sociedad, porque la mayor morbilidad que presenta está influida por factores derivados de la manera en que se integran al mercado del trabajo, a espacios de participación ${ }^{1,2}$ y por un mayor estrés e infelicidad que se derivan de condiciones de vida con más vulnerabilidad social ${ }^{3-6}$.

La visión de la salud desde el género es imprescindible para el diseño e implementación de políticas públicas de salud, no sólo por un imperativo ético de equidad, sino también porque el género condiciona el perfil epidemiológico de la salud de las personas, el logro de objetivos sanitarios y la calidad y eficiencia de las prestaciones de salud $3,4,7-11$

Los estudios epidemiológicos documentan diferencias de género en la prevalencia de diversas enfermedades ${ }^{4,12-15}$. Las estadísticas de salud mental muestran que las mujeres presentan un índice más alto de trastornos mentales que los hombres ${ }^{5,6}$, hecho que traduce presiones psicosociales a las que están sometidas y a la predisposición de la sociedad patriarcal para clasificar a las mujeres como enfermas mentales ${ }^{5,6,16,17}$.

Las relaciones de género inciden y son afectadas por el modelo de atención en salud ${ }^{18,19}$. Porque en las prácticas cotidianas de atención emergen los estereotipos tradicionales de género ${ }^{17}$. Las mujeres consultantes tienden a presentar sus casos relacionando sus síntomas con diversos aspectos de su vida diaria, así como con sus propias opiniones y creencias a propósito de lo que les pasa16,18. Las y los profesionales de la salud enfatizan la objetividad y la separación entre el sujeto que conoce y los objetos que son conocidos, tienden a dirigir la conversación en términos estrictamente clínicos, sin permitir que las mujeres se expresen como ellas desean. Entre prestatarios y usuarias existe una relación jerárquica asimétrica que se agudiza en el caso de mujeres de estratos sociales bajos, situación que explica muchos de los desencuentros que se producen $16,18,19$

Otros aspectos que complementan el análisis para el sector salud son los costes invisibles de la enfermedad $^{20}$ y los efectos de la segregación sexual respecto a la calidad de los empleos por género, en las condiciones y responsabilidades de trabajo, el acceso a los cargos de decisión, acceso a desarrollo profesional, los ingresos y la calidad de la protección y apoyo social ${ }^{21-26}$.

El género es una categoría transversal, de relación social y política, que opera en múltiples niveles y que afecta cotidianamente las interacciones y comportamientos sociales ${ }^{16}$. Asegurar la salud como derecho humano universal involucra una co-responsabilidad social de la educación superior en la formación de recursos humanos con perspectiva de género $8,27-29$, por tanto, la educación superior debe implementar una concepción pedagógica, epidemiológica y social de la salud con perspectiva de género, para comprender el vínculo entre la biología con la dimensión social y los significados culturales de las personas $^{17,30}$

La declaración mundial de la educación superior en el siglo $\mathrm{XXI}^{28}$, señala que la universidad tiene la misión de: i) establecer un sistema de educación superior equitativo y no discriminatorio, fundado en el principio del mérito; ii) eliminar todos los estereotipos fundados en el género en la educación superior; iii) tener en cuenta el punto de vista del género en las distintas disciplinas; iv) consolidar la participación cualitativa de las mujeres en todos los niveles y las disciplinas en que están insuficientemente representadas; v) incrementar su participación activa en la adopción de decisiones y vi) responder a las demandas sociales con innovaciones y pertinencia en el diseño de prácticas educativas vinculadas con comportamientos y cambios sociales.

El curículo explícito (formal u oficial) hace referencia al documento escrito que establece el enfoque y estrategias pedagógicas, sus objetivos, contenidos, metodología, recursos, y criterios de evaluación, etc ${ }^{31}$. A los efectos, el programa asignatura, fue valorado y conceptuado como el discurso pedagógico explícito, regulador simbólico de la selección, creación, posición y oposición de sujetos pedagógicos para la producción, reproducción y transformación de la cultura institucional de género. El currículo obviado hace referencia a un conjunto de situaciones que se presentan en los procesos de enseñanza aprendizaje, y que se concretan en el perfil docente y rendimiento estudiantil.

Bajo estos considerandos, se realizó un estudio exploratorio descriptivo con el objetivo de identificar el género y perspectiva de género en el currículo explícito de cinco carreras de la salud de 
la Facultad de Medicina, Universidad Austral de Chile, período 2003-2004, e identificar en el currículo obviado la situación de las mujeres en el perfil docente y rendimiento académico de estudiantes.

\section{MATERIAL Y MÉTODO}

Tipo de estudio. Se realizó un estudio exploratorio, que articuló un diseño descriptivo bajo procedimientos cuantitativos. La unidad de análisis fue, en una primera fase, el discurso pedagógico programas de asignaturas- de cinco carreras: Enfermería, Medicina, Obstetricia y Puericultura, Tecnología Médica y Terapia Ocupacional, 20032004. En la segunda fase, se recabó información desde el Sistema de Información Curricular (SIC) de la situación de hombres y mujeres docentes y estudiantes en el proceso pedagógico en el período señalado.

Criterio de inclusión. El criterio de inclusión fue que las asignaturas estuvieran inscritas en el SIC, con información sobre número y rendimiento académico de estudiantes, identificación, carga académica y condición laboral del docente responsable de la asignatura.

Recolección de la información. El análisis de documentos fue realizado por una investigadora para asegurar uniformidad en los criterios definidos para la lectura crítica. La información sobre variables de docentes y estudiantes fue obtenida por el equipo de investigación desde: programas, base de datos del registro académico oficial, base de datos de Dirección de Personal y del Departamento de Matrículas.

Mediciones y procedimientos. Primera fase, se realizó análisis crítico de contenidos curriculares descritos en el discurso pedagógico, formal y escrito, con identificación de descriptores curriculares y temas transversales adicionales. Se usó una lista de cotejo con variables relacionadas con lenguaje genérico -como recurso inclusivo o de exclusión- en programas básicos, preprofesionales, profesionales, optativos y facultativos. Se buscó especificidades de género y perspectiva de género en: i) competencias básicas, técnicas y transversales explícitas en la estructura del programa; ii) objetivos generales, objetivos específicos, unidades temáticas y contenidos; iii) metodologías, materiales bibliográficos, métodos didácticos, planeamiento de prácticas y ejercicios educativos orientadas a modificar pautas culturales que diferencian habilidades, conocimientos y destrezas de manera diferenciada, para hombres y mujeres, incluidos en espacios de aprendizaje común; iv) en la identificación de género en docentes responsables de asignatura. La medición se hizo en escala formato Likert (siempre, frecuentemente, a veces, rara vez, nunca). Para medición de variables sobre metodología se usó escala binaria (presente, ausente). Se incluyó variables adicionales sobre competencias transversales: trabajo colaborativo, relación interpersonal, relación democrática, equidad y análisis crítico.

Segunda fase: La situación de docentes, hombres y mujeres, fue contextualizada con variables sobre carga académica y laboral, tipo de contrato, categoría académica y sexo de docentes responsables de asignatura. Para estudiantes se recabó información por asignaturas sobre: número de estudiantes según sexo y rendimiento de estudiantes por sexo y carrera (resultado académico respecto a notas promedio finales y porcentaje de reprobación de estudiantes por asignaturas y carrera).

Estadística. Se configuraron bases de datos brutos y depurados. Se calcularon estadígrafos descriptivos de frecuencia, posición y dispersión por carreras y asignaturas. Para la comparación de variables ordinales y nominales se usó estadísticas Chi cuadrado, Coeficiente de Phi (para tablas $2 \mathrm{x} 2$ ), $\mathrm{V}$ de Cramer (tablas kxn) y para variables discretas t de Student. Se estableció un nivel de confianza de $95 \%$. Se usó los programas computacionales S.P.S.S. y Epi Info versión 6.

\section{Resultados}

Del universo de 245 asignaturas ejecutadas en su última versión, $217(88,6 \%)$ cumplieron con el criterio de inclusión, (Figura 1a). El material revisado corresponde en $99,5 \%$ a asignaturas semestrales; del ciclo básico (34,7\%); preprofesional (23,6\%); profesional (38,0\%) y optativas 


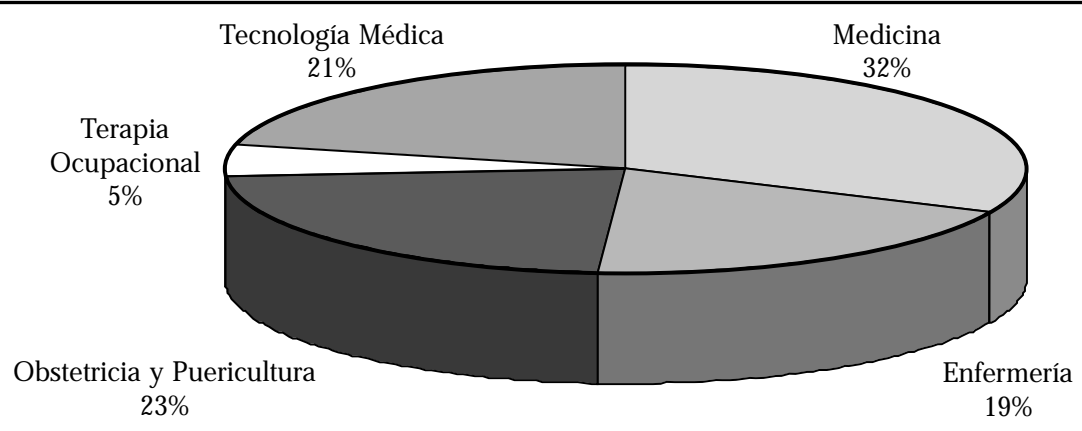

Figura 1a. Distribución numérica de 217 asignaturas revisadas por carreras de la Facultad de Medicina, Universidad Austral de Chile, 2003-2004.

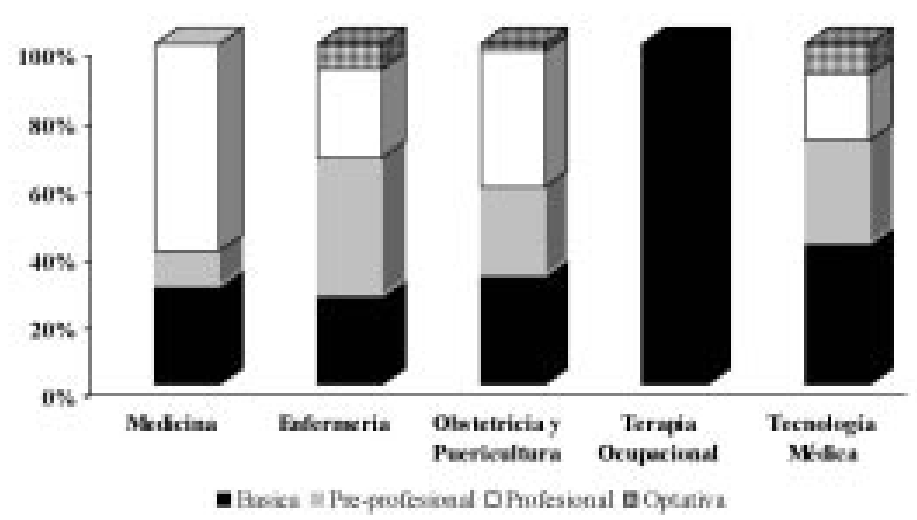

Figura 1b. Distribución porcentual asignaturas currículo básico, preclínico y profesional, por carreras de la Facultad de Medicina, Universidad Austral de Chile, período 2003-2004.

(3,7\%); dictadas en última versión en el primer $(43,1 \%)$ o segundo semestre (56,9\%). La distribución por carrera se presenta en la Figura 1b. La menor proporción de asignaturas para Terapia Ocupacional se debe a que la malla curricular se inició en el año 2004, y a la fecha en que se recaba la información no se habían dictado asignaturas pre-profesionales y profesionales.

Primera fase. En 82,9\% de los programas no se utilizó un lenguaje diferencial de género para estudiantes (nunca $=82,9 \%$ ). Tampoco se explicitó en la descripción del programa el efecto diferencial de la disciplina en la población femenina y masculina (nunca $=95,4 \%$ ). En la estructura pedagógica no fue incorporada la perspectiva de género en: i) los objetivos generales (nunca $=96,3 \%$ ) y específicos (nunca $=94,5 \%$ ); ii) compe- tencias básicas, técnicas y transversales (nunca $=95,4 \%$; y iii) la presentación de los contenidos, la promoción del análisis de información, temas de estudio y bibliografía desagregada por género (nunca $=94,9 \%$ ) (Figura 2). Estuvo ausente la incorporación de perspectiva de género en: i) la selección de métodos que consideran las percepciones de hombres y mujeres sobre la iniciativa, el compromiso con el mejoramiento continuo, la comunicación efectiva, liderazgo, toma de decisiones $(99,1 \%) \mathrm{y}$, ii) la selección de prácticas y ejercicios educativos dirigidas a modificar pautas culturales genéricas (estereotipos) que diferencian habilidades, conocimientos y destrezas (100\%). De igual manera, prevaleció la ausencia total de oferta de espacios que estimulen el ejercicio de liderazgo de hombres y mujeres en igualdad 


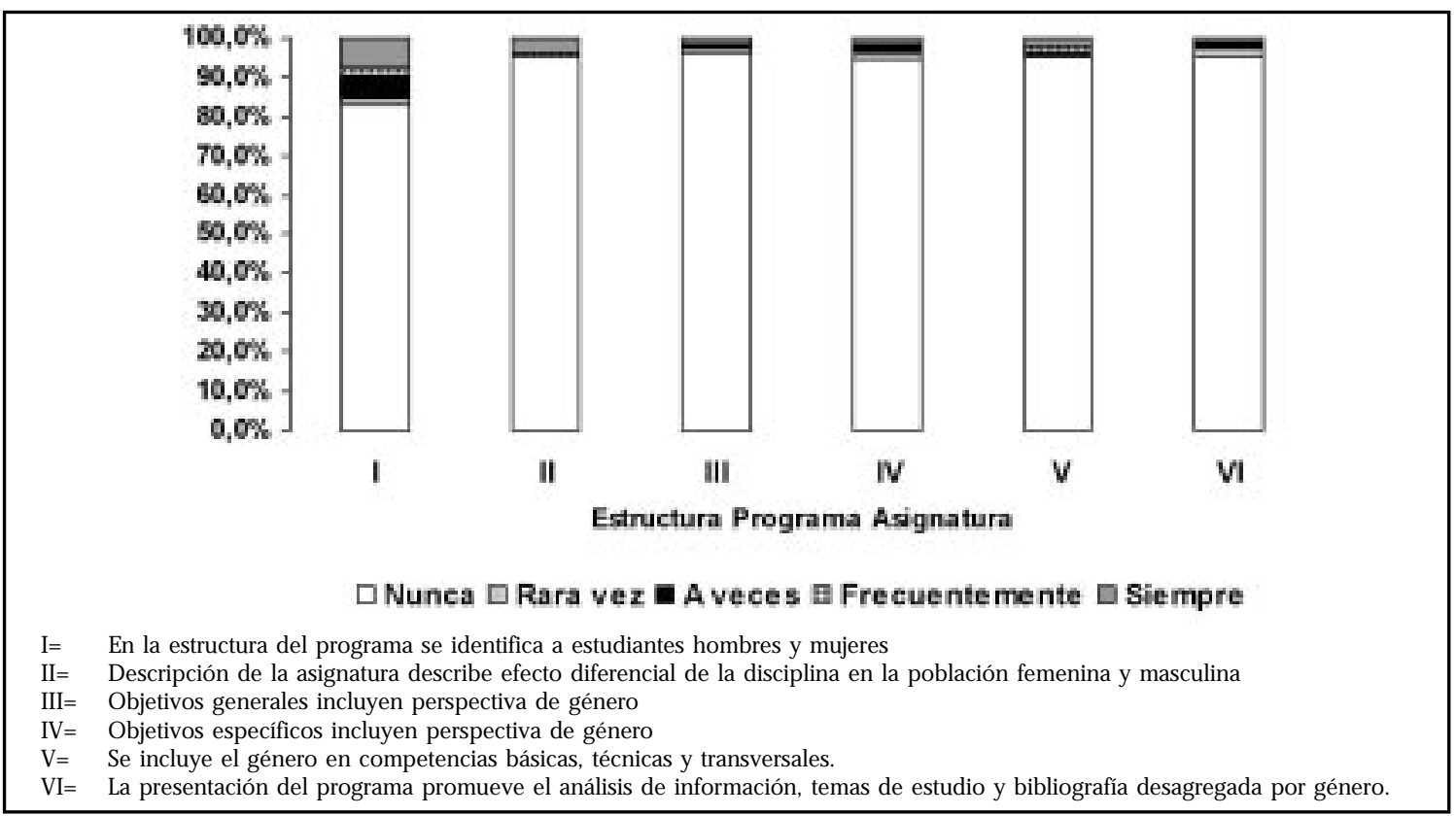

Figura 2. Distribución porcentual de inclusión de perspectiva de género en estructura de los programas, Facultad de Medicina, Universidad Austral de Chile, período 2003-2004.

(100\%). No se utilizó un lenguaje genérico inclusivo para hombres y mujeres en el discurso pedagógico de la asignatura (nunca $=81,1 \%$ ). A lo anterior, se agrega que en la bibliografía no se especificó la identificación completa de autoría y, cuando se hizo, las mujeres nunca y rara vez son citadas como autoras en la bibliografía recomendada. El análisis por carrera mostró similar comportamiento. Sobre los resultados de la inclusión de competencias transversales en la estructura de los programas, se encontró que nunca se explicitaron orientaciones sobre: trabajo colaborativo $(86,6 \%)$, relación interpersonal $(80,2 \%)$, relación democrática $(99,1 \%)$, equidad $(96,8 \%)$ y análisis crítico $(65,4 \%)$, que por carreras no difiere, especialmente, en asignaturas profesionales (Figura 3).

Segunda Fase. Mujeres académicas: $54,4 \%$ de las asignaturas tenía una mujer como docente responsable y en una por cada cinco asignaturas se explicitó su identidad de género, situación que en académicos hombres se observó en $90 \%$ de las asignaturas. Similares registros se encontró por carrera con excepción de asignaturas de Terapia Ocupacional (Figura 4). A esta invisibilidad, se suman los datos sobre la categoría académica de docentes responsables de asignaturas que evidencian que la mayor parte de las mujeres tenían categoría académica instructora y auxiliar y los hombres categoría académica de profesor titular y asociado (Figura 5), p <0,05. En las asignaturas de las carreras de Medicina y Tecnología Médica el perfil de docentes responsables de las asignaturas fue más homogéneo.

Mujeres estudiantes: La invisibilidad de estudiantes mujeres en el discurso pedagógico explícito se revierte en los registros del SIC en el total de las asignaturas revisadas. Se encontró una relación de 1 hombre por cada 2 mujeres, relación que aumentó a favor de estudiantes mujeres en asignaturas de las carreras de Enfermería (6:1), Obstetricia y Puericultura y Terapia Ocupacional (4:1). En las carreras de Medicina y Tecnología Médica la relación fue más homogénea (1:1 y 1:1,3 respectivamente).

La información sobre rendimiento académico, medido por calificaciones promedio finales y proporción de reprobación por sexo, mostró que estudiantes mujeres obtuvieron mejores calificaciones que los estudiantes hombres en asignaturas de las carreras de Medicina, Enfermería y Obstetricia y 


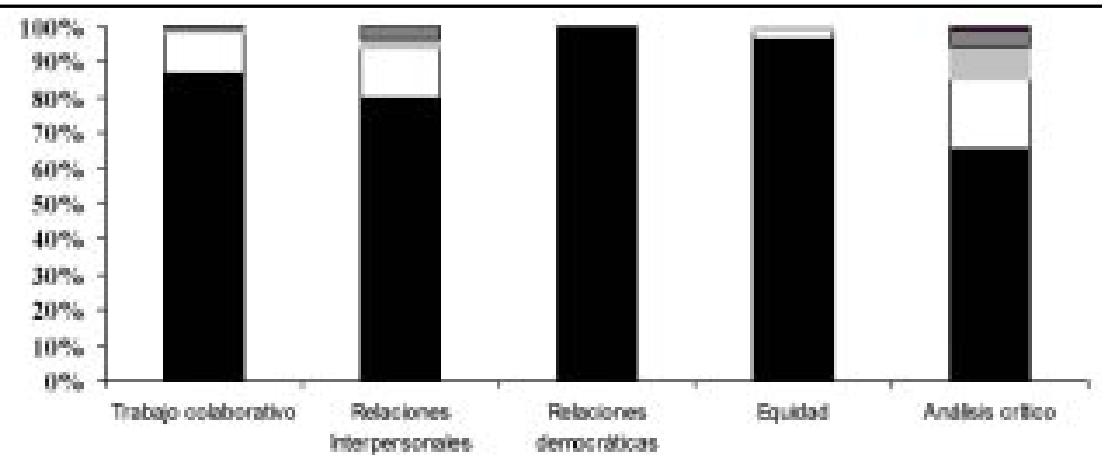

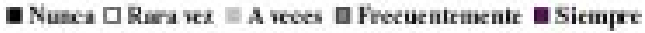

Figura 3. Distribución porcentual de inclusión de temas transversales en asignaturas de cinco carreras de la salud, Facultad de Medicina, Universidad Austral de Chile, 2003-2004.

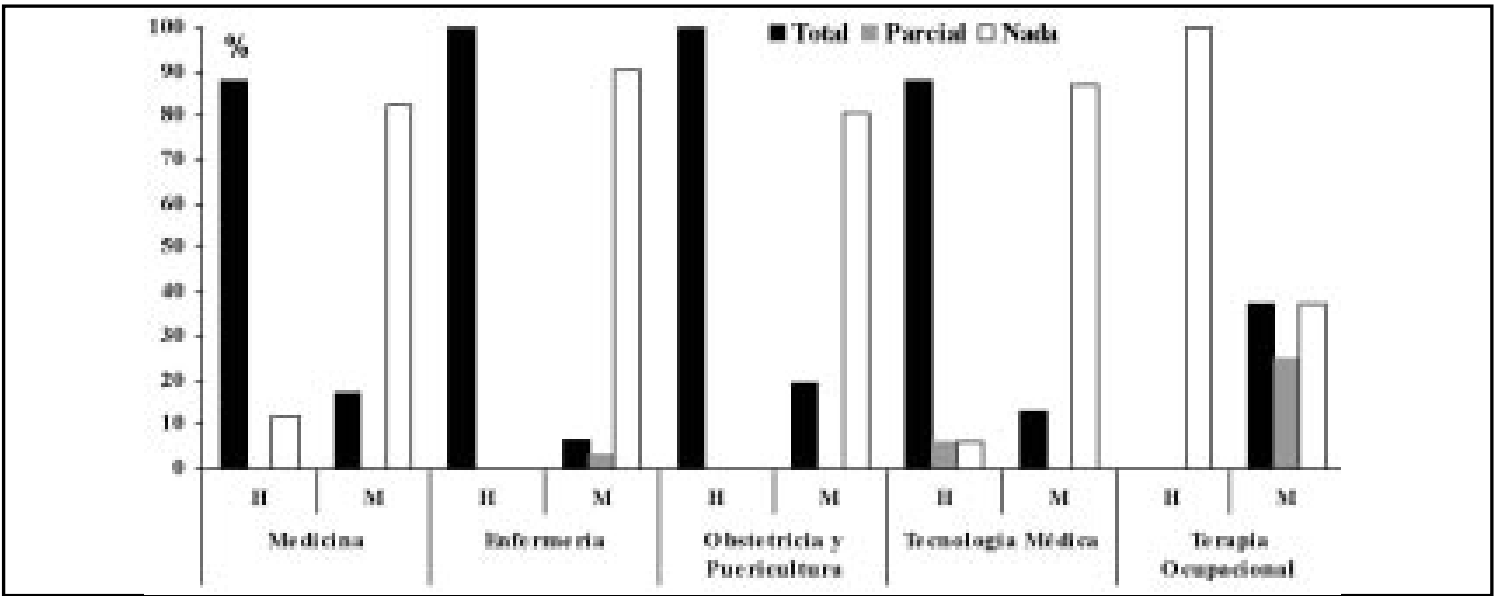

Figura 4. Distribución porcentual de inclusión de identidad de género de docentes responsables de las asignaturas, por carreras de la Facultad de Medicina, Universidad Austral de Chile, período 2003-2004.

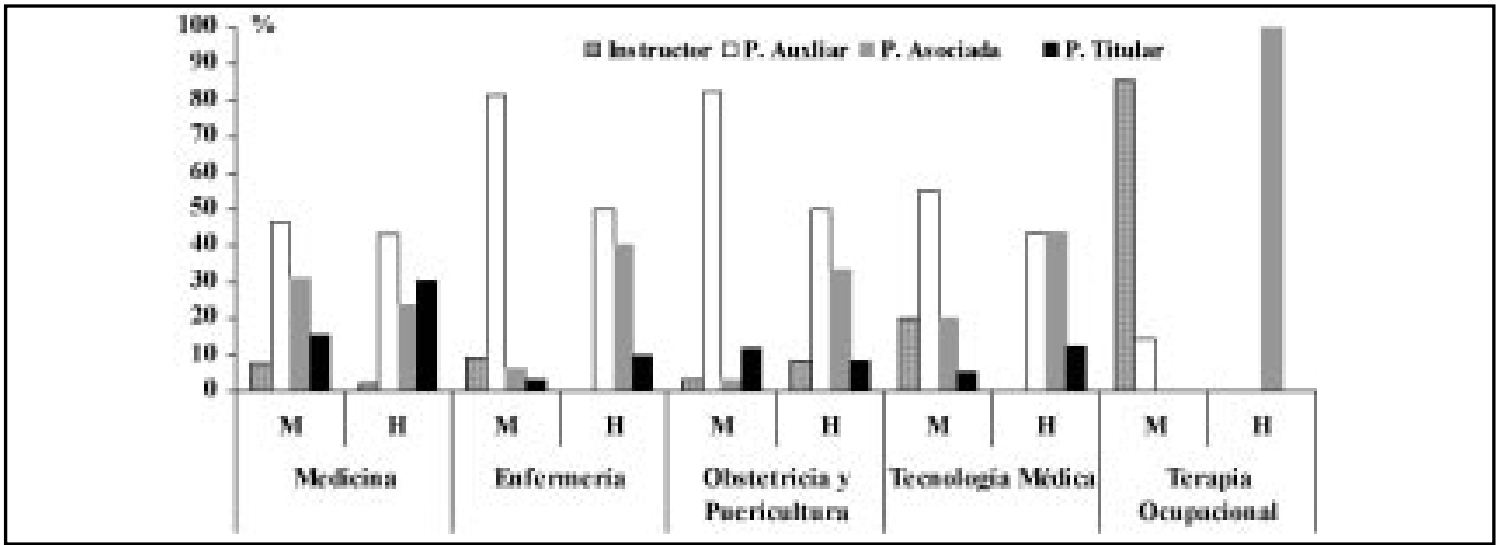

Figura 5. Distribución porcentual de asignaturas por categonía académica* registrada, según género docente responsable, y por carreras de la Facultad de Medicina, Universidad Austral de Chile, período 2003-2004. *Categorías académicas: I Profesor Titular, II Profesor Asociado, III Profesor Auxiliar e Instructor. 
Puericultura, situación que se revierte en asignaturas de Terapia Ocupacional (Figura 6). El porcentaje de asignaturas reprobadas según sexo, fue más alta en estudiantes hombres en asignaturas de las carreras de Medicina y Terapia Ocupacional. Las mujeres reprueban más en asignaturas de la malla curricular de las carreras de Enfermería, Obstetricia y Puericultura y Tecnología Médica (Tabla 1).

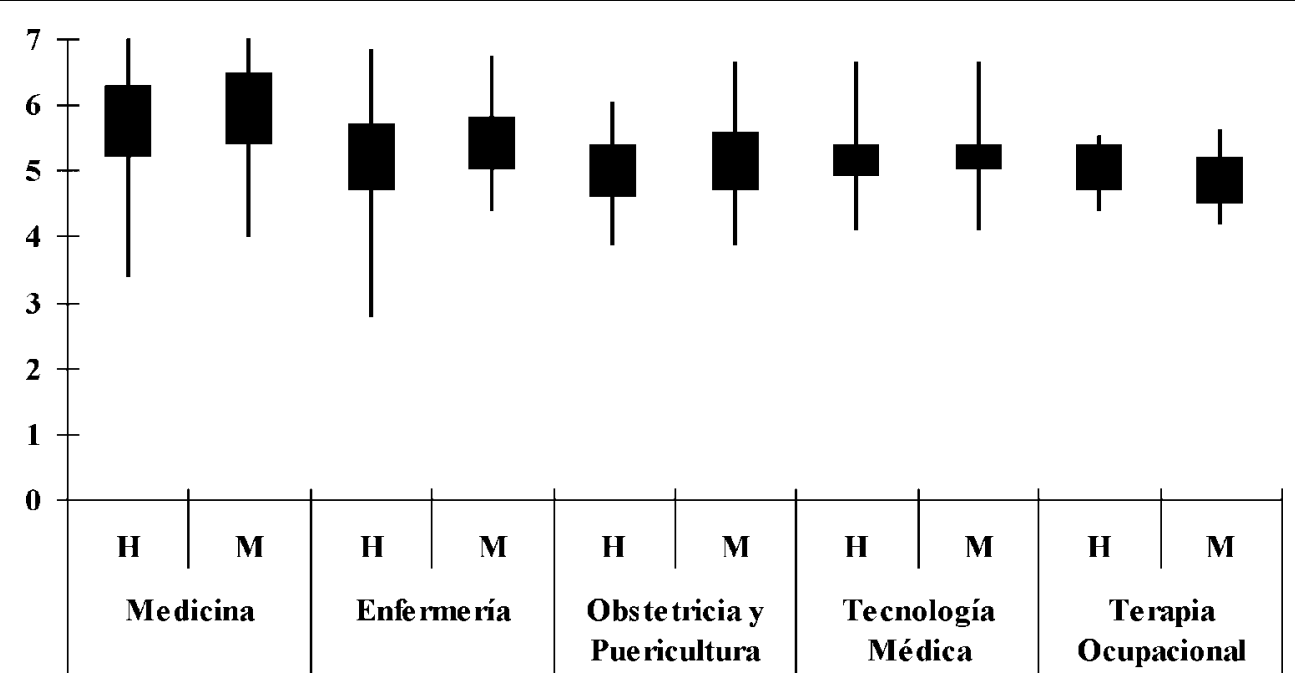

* Los box-plot muestran la posición de la nota promedio mínima, la del percentil 25, la del percentil 75 y la nota máxima.

Figura 6. Distribución notas* de asignaturas revisadas por carreras, según género estudiante, Facultad de Medicina, Universidad Austral de Chile, 2003-2004.

Tabla 1. D istribución porcentual de asignaturas reprobadas por carreras según género estudiantes, Facultad de Medicina, Universidad Austral de Chile, 2003-2004

\begin{tabular}{|llrrrr|}
\hline Carrera & \% asignaturas reprobadas & \multicolumn{2}{c}{ Hombres } & \multicolumn{2}{c|}{ Mujeres } \\
& & $\mathrm{N}^{\circ}$ & $\%$ & $\mathrm{~N}^{\circ}$ & $\%$ \\
\hline Medicina & $<1 \%$ & 42 & 62,7 & 56 & 83,6 \\
(n= 67 asignaturas) & $1 \%-5 \%$ & 12 & 17,9 & 7 & 10,4 \\
& Más de 5\% & 13 & 19,4 & 4 & 6,0 \\
Enfermería & $<1 \%$ & 35 & 87,5 & 25 & 59,5 \\
(n= 42 asignaturas & $1 \%-5 \%$ & & & 11 & 26,2 \\
muj 40 ho) & Más de 5\% & 5 & 12,5 & 6 & 14,3 \\
Obstetricia y & $<1 \%$ & 32 & 68,1 & 32 & 64,0 \\
Puericultura & $1 \%-5 \%$ & & & 3 & 6,0 \\
& Más de 5\% & 17 & 34,6 & 15 & 30,0 \\
Tecnología Médica & $<1 \%$ & 35 & 77,8 & 34 & 75,6 \\
& $1 \%-5 \%$ & 2 & 4,4 & 2 & 4,4 \\
Terapia & Más de 5\% & 8 & 17,8 & 9 & 20,0 \\
Ocupacional & $<1 \%$ & 14 & 45,2 & 16 & 51,6 \\
& $1 \%-5 \%$ & 11 & 35,5 & 9 & 29,0 \\
& Más de 5\% & 6 & 19,3 & 6 & 19,4 \\
\hline
\end{tabular}




\section{DisCUSIÓN}

El enfoque de género incorpora un marco conceptual que permite descifrar la realidad social a través de la epidemiología de las diferencias y promueve el desarrollo de competencias profesionales para la interpretación desagregada de los problemas de salud ${ }^{11,32}$. De esta forma, se podrá develar las inequidades de salud, definir perfiles epidemiológicos de género y formular diagnósticos con prácticas sanitarias con perspectiva de género ${ }^{4,19,32-34}$.

En Chile sigue pendiente la incorporación de perspectiva de género en la formación de recursos humanos ${ }^{35}$. Hasta ahora, la aproximación de las universidades chilenas ha sido a través de la investigación y estudios de posgrado. No hemos encontrado evidencias de incorporación del género como eje transversal en las mallas curriculares de estudios de pregrado ${ }^{35}$.

Sobre el currículo obviado, los resultados muestran un discurso pedagógico con lenguaje sexista que invisibiliza la participación de mujeres académicas y estudiantes, con falta de autorreconocimiento de las propias mujeres como parte de la dotación académica de las asignaturas ${ }^{27}$, es decir, existe una práctica discriminatoria que pasa inadvertida para hombres y mujeres ${ }^{16,17,36}$. Desde hace tiempo se insiste que es una cuestión de voluntad explicitar que las mujeres ocupan espacios, realizan funciones $\mathrm{y}$, por tanto, tienen que nombrarse ${ }^{36,37}$. Los datos sobre el perfil contractual descrito para la mujer académica reproducen la «natural»desigualdad de género que se reconoce en el mercado del trabajo $1,21,27$.

La preeminencia de estudiantes mujeres en carreras de la salud representa el significado del aprendizaje social de los roles tradicionales de

\section{REFERENCIAS}

1. AвRAmo L Trabajo decente y equidad género en América Latina. Oficina Internacional del Trabajo, O.I.T., 2006, 316p. Disponible en: http:// www. cinterfor.org.uy/public/spanish/region/ ampro/cinterfor/new sro o m/resenas/2006/ tdec_gen.pdf. Consultado el 5 de agosto 2006. género en la elección de la profesión ${ }^{35,36,38}$. Las diferencias encontradas respecto al rendimiento académico en notas promedio finales y reprobación de asignaturas según género no dan cuenta de una diferencia radical y absoluta ${ }^{39}$, hallazgo que refuerza nuestro cuestionamiento sobre la discriminación que se observa en la academia y que, posteriormente, se reproduce en el mercado laboral.

El escenario académico descrito genera una visión poco optimista sobre las posibilidades de un cambio cultural generado desde las universida$\operatorname{des}^{29}$. Por el contrario, la universidad se constituye en un peldaño más en la escala de la socialización de los sujetos donde las ideas del sistema patriarcal se forman, legitiman y reproducen 29,38 . Como consecuencia, no hay sintonía con los cambios que requiere la sociedad chilena para asegurar a las personas equidad en salud 4 , nodiscriminación en el acceso a las oportunidades y una convivencia social democrática ${ }^{27,38,34}$. Tampoco se garantiza la formación de recursos humanos con capacidad de respuesta coherente a las nuevas tendencias epidemiológicas y diversidad de necesidades desagregadas por género $27,32,34,40$.

La reforma educacional chilena promueve una educación superior de calidad ${ }^{38}$ y con pertinencia social, por tanto, la integración del género es un desafío y exigencia inaplazable en todos los aspectos de política y diseño curricular en la educación superior en salud ${ }^{28,32}$. No asumir el desafío significa preservar la medicalización de la atención de salud, mantener los niveles de inequidad e insatisfacción de las personas, continuar con altos índices de policonsulta $\mathrm{y}$, también, conservar sesgos y segregaciones que se gestan en la educación superior y se consolidan en el mercado laboral ${ }^{1,4,35,38}$.

2. ZÚNIIGA Y. Democracia paritaria: De la teoría a la práctica. Rev Derecho (Valdivia) 2005; 18: 131-54.

3. Behar R, De la Barrera M, Michelotti J. Feminidad, masculinidad, androginidad y trastornos del hábito del comer. Rev Méd Chile 2002; 130: 964-75.

4. Vega J, Bedregal P, Jadue L, Delgado I. Equidad de género en el acceso a la atención de salud en Chile. Rev Méd Chile 2003; 131: 669-78. 
5. Cova F, Valdivia M, Maganto C. Diferencias de género en psicopatología en la niñez: Hipótesis explicativas. Rev Chil Pediatr 2005; 76: 418-24.

6. WHO Women's mental health: An evidence based review. Mental Health Determinants and Populations, Departamental of Mental Health and Substance Dependence, World Health Organization, Geneva 2000. Disponible en: <http://www. who.int/mental_health/resources/gender/en/ index.html). Consultado en abril 2006.

7. JEWKes R. A prescription for gender, health and human rights. Lancet 2004; 363: 91.

8. Provoste P. Participación en la Salud Pública: Una aproximación de género. En: Género, equidad y reforma de la salud en Chile. Serie Género y Reforma de la Salud, Organización Panamericana de la Salud y Organización Mundial de la Salud 2002. Disponible en: http://www.ops-oms.org/ Spanish/DPM/GPP/GH/Cuadernillo2.pdf. Consultado en febrero 2006.

9. Theobald S, Elsey H, Tolmurst R. Gender, health and development I: gender equity and sector wide approaches. Prog Dev Stud 2004; 4: 58-63.

10. Szot Meza J. Mortalidad por infarto agudo al miocardio en Chile: 1990-2001. Rev Méd Chile 2004; 132: 1227-33.

11. BEKKER J. Investigating gender within health research is more than sex disaggregation of data: a Multi-Facet and Health Model. Psychol Health Med 2003; 8: 231-43.

12. Castro-Sansores C, Santos-Rivero A, Lara-Perera D, Gonzáles-Martínez P, Alonso-Salomón G, GóngoraBIACHI R. Hiperlipidemia e intolerancia a la glucosa en un grupo de pacientes infectados con VIH que reciben terapia antirretrovírica hiperactiva. Salud Pública Méx 2006; 48: 193-9.

13. Herrera C, Campero L La vulnerabilidad e invisibilidad de las mujeres ante el VIH/SIDA: constantes y cambios en el tema. Salud Pública Méx 2002; 44: 554-64.

14. Olveira R. Gênero, direitos humanos e impacto socioeconômico da Aids no Brasil. Rev Saúde Pública 2006; 40: 80-7.

15. Marín-León L, BarRos M. Mortes por suicídio: diferenças de gênero e nível socioeconômico. Rev Saúde Pública 2003; 37: 357-63.

16. Castro R, Bronfman M. Teoría feminista y sociología médica: bases para una discusión. Cad Saúde Pública 1993; 9: 375-94.

17. AraYA C. La construcción de una imagen femenina a través del discurso médico ilustrado: Chile en el siglo XIX. Historia (Santiago) 2006; 39: 5-22.

18. Cuesta-BenjuRnea C. Las mujeres y el manejo de un síntoma: De la valoración a la selección. Salud Pública Mex 1999; 41: 124-9.

19. Provoste P, Berlagoscky F. Modelo de atención y género: Las condiciones socioculturales de la reforma de la salud en Chile. Género, equidad y reforma de la salud en Chile. Organización Panamericana de la Salud y Organización Mundial de la Salud. 2002. Disponible en: http://www.opsoms.org/Spanish/DPM/GPP/GH/Cuadernillo2.pdf. Consultado en febrero 2006.

20. DuRán MA. Los costes de la salud para las familias. En Apuntes desde el género para una economía de la salud. Género, Equidad y Reforma de la salud en Chile. Organización Panamericana de la Salud y Organización Mundial de la Salud, 2002. Disponible en: http://www. generoreforma.org/Cuadernillo3.pdf. Consultado en febrero 2006.

21. Papadópulos J, Radakovich R. Educación Superior y Género en América Latina y el Caribe. Estudios y documentos del Consejo Superior de Educación de Chile, 2006. Disponible en: http://www.cse.cl/public/ Secciones/seccionestudios/estudios_y_documentos de Genero.aspx. Consultado en agosto 2006.

22. CASTRO $R$, CAMPERO L, HeRnández B. La investigación sobre apoyo social en salud: situación actual y nuevos desafíos. Rev Saúde Pública 1997; 31: 425-35.

23. Trucco M, Valenzuela P, Trucco D. Estrés ocupacional en personal de salud. Rev Méd Chile 1999; 127: 1453-61.

24. Meneghel S, Barbiani R, StefFen $H$, Wunder A, Daila Rosa M, Rotermund J et al. Impacto de grupos de mulheres em situação de vulnerabilidade de gênero. Cad Saúde Pública 2003; 19: 955-63.

25. OPS-OMS. Discriminación de las mujeres en el sistema de instituciones de salud previsional. Regulación y perspectiva de género en la reforma. Género, Equidad y Reforma de la salud en Chile. Organización Panamericana de la Salud y Organización Mundial de la Salud, 2002. Disponible en: http://www.generoreforma.org/Cuadernillo1.pdf. Consultado en abril 2006.

26. Hitlan R, Cuffton R, Desoto C. Perceived exclusion in the workplace: The moderating effects of gender on work-related attitudes and psychological health. North American Journal of Psychology 2006; 8: 217-36. 
27. Oyarzún K. Un trato en las diferencias: Género y educación superior en Chile. Estudios y documentos del Consejo Superior de Educación de Chile. 2005. Disponible en: http://www.cse.cl/ public/Secciones/seccionestudios/estudios_y_documentos_de_Genero.aspx. Consultado en octubre 2006.

28. UNESCO. Artículo 4. Fortalecimiento de la participación y promoción del acceso de las mujeres. Declaración mundial sobre la educación superior en el siglo XXI: visión y acción preámbulo. Aprobada por la Conferencia Mundial sobre la educación superior, realizada en Paris 1998. Disponible en: http://www.unesco.org/cpp/sp/declaraciones/ world.htm. Consultado el 18 de agosto 2006.

29. Arcos E, Molina I, Trumper RE, Larrañaga L, Del Río MI, Tomic P et al. Percepción del género y perspectiva de género en estudiantes y docentes de la Universidad Austral de Chile. Estud Pedagóg 2006; 32: 33-47.

30. Roter D, HaLl J. Physician gender and patientcentered communication: A critical review of empirical research. Annu Rev Public Health 2004; 25: 497-19.

31. Соокs L, Sun CH. Constructing gender pedagogies: Desire and resistance in the alternative classroom. Communication Education 2002; 53: 293-310.

32. Castro R. Género y política en salud. Salud Pública Méx 2003; 45: 419-21.

33. Pошаск M. Equidad de género en el sistema de salud chileno. Serie Financiamiento del Desarrollo 2002; 123: 1-43. Disponible en: http://www.eclac.cl/publicaciones/Comercio/4/LCL1784P/lcl1784e.pdf. Consultado en abril 2006.

34. OPS-OMS. Observatorio de equidad de género en salud. Informe 2005. Proyecto Género, equidad y reforma, Chile, Segunda Fase. Disponible en: http:// pwr-chi.bvsalud.org/dol/docsonline/ get.php?1d=226. Consultado el 5 de agosto 2006.

35. RoJAs T. La educación superior en Chile durante los últimos 25 años: una aproximación de género. Observatorio digital para la educación superior del Instituto Internacional para la Educación Superior para América Latina y el Caribe (IESALC). Organización de las Naciones Unidas para la Educación, Ciencia y Cultura, 2003. Disponible en: http://www.iesalc.unesco.org.ve/programas/ GENERO/GéneroChile.pdf. Consultado en octubre 2006.

36. VICENTY C. Socialización, lenguaje y educación: Una mirada desde el enfoque de género. RAE 2003; 3: 1-3. Disponible en: http://www.ujaen.es/ huesped/rae. Consultado el 9 de octubre 2006.

37. CONICYT-Chile. Mujeres en ciencias y tecnología. Lenguaje y género. Programa de Mejoramiento de la Gestión (PMG) de Género, Consejo Nacional de Investigación y Tecnología de Chile. Disponible en: http:// www.conicyt.cl/genero/avances/ lenguaje_genero.html. Consultado en octubre 2006.

38. REYES J. Trabajadoras (es) de la educación superior y reproducciones de género. Estudios y documentos del Consejo Superior de Educación de Chile, 2006. Disponible en: http://www.cse.cl/ public/Secciones/seccionestudios/estudios_y_documentos de_Genero.aspx. Consultado en octubre 2006.

39. Paulus N, Griggs T. Proceso de examinación del CSE: Incidencia de la variable género en el rendimiento académico. Estudios realizados por la Secretaría Técnica, Consejo Superior de Educación, 2004. Disponible en: http://www.cse.cl/ public/Secciones/seccionestudios/estudios_y_documentos_de_Genero.aspx. Consultado en octubre 2006.

40. DAY A. Lessons from the women's health initiative: primary prevention and gender health. CMAJ 2002; 167: 361-2. 